\title{
DECOMPOSITION OF APPROXIMATE DERIVATIVES
}

\author{
RICHARD J. O'MALLEY ${ }^{1}$
}

\begin{abstract}
It is shown that if $f:[0,1] \rightarrow R$ has a finite approximate derivative $f_{\text {ap }}^{\prime}$ everywhere in $[0,1]$, then there is a sequence of perfect sets $H_{n}$, whose union is $[0,1]$, and a sequence of differentiable functions, $h_{n}$, such that $h_{n}=f$ over $H_{n}$ and $h_{n}^{\prime}=f_{\text {ap }}^{\prime}$ over $H_{n}$. This result follows from a new, more general theorem relating approximate differentiability and differentiability. Applications of both theorems are given.
\end{abstract}

In this paper we illustrate a new sense in which an approximate derivative is a derivative. This approach can be used to both clarify known properties of these derivatives and also establish additional properties. Basically, we prove that an approximate derivative can be decomposed, in a way which will be made precise, into a sequence of derivatives. This result will be obtained from the following new theorem relating the concepts of differentiability and approximate differentiability.

THEOREM 1. Let $Q$ be a measurable set and $E$ a closed subset of the points of density of $Q$. Suppose $f: Q \rightarrow R$ is a measurable function possessing a finite approximate derivative at each point of $E$. Then $E$ can be expressed as the countable union of closed sets $E_{n}$ such that for each $n$ and each $x$ in $E_{n}$,

$$
E_{n}-\lim _{y \rightarrow x} \frac{f(y)-f(x)}{y-x}=f_{\text {ap }}^{\prime}(x) .
$$

Here the notation $E_{n}-\lim _{y \rightarrow x}$ means that we approach $x$ only through the set $E_{n} \backslash\{x\}$. At an isolated point of $E_{n}$ the conclusion is considered to hold vacuously. It will be shown later that the sets $E_{n}$ can be chosen to be perfect sets.

Before the proof we state a lemma. It is a modification of lemmas which can be found in [4] and [5].

Lemma. Let $Q$ and $E$ be as stated in Theorem 1. Suppose $f$ is approximately continuous at every point of $E$. Let $n$ be a fixed integer. Define

$$
A_{n}(x)=\{y \in Q:|f(y)-f(x)| \leqslant n|y-x|\}
$$

and

Received by the editors July 11, 1977.

AMS (MOS) subject classifications (1970). Primary 26A24; Secondary 26A21.

Key words and phrases. Points of density, approximate derivative, Baire category theorem.

${ }^{1}$ Author supported in part by a research grant from the University of Wisconsin-Milwaukee. 


$$
E_{n}=\left\{x \in E: m\left(A_{n} \cap I\right) \geqslant \frac{3}{4} m(I)\right. \text { for all intervals }
$$

I containing $x$ with length of $I<1 / n\}$.

(Here $m$ denotes Lebesgue measure.) Then

(a) $E_{n}$ is a closed set, and

(b) if $x$ and $y$ belong to $E_{n}$ and $|x-y|<1 / n$, then $|f(x)-f(y)| \leqslant n|x-y|$.

Proof. The proof of (b) is obvious. The proof of (a) is rather lengthy and requires proving first that (b) holds at every two limit points of $E_{n}$. Since the proof can be arrived at by modifying appropriately the proofs in [4] or [5] it will not be given here. We proceed instead with the proof of Theorem 1 .

Proof of TheOrem 1. For each $n$ we apply the above Lemma to get a sequence of closed sets $E_{n}$. These $E_{n}$ form the desired decomposition of $E$. To see this let $x$ belong to $E$. There is an $N_{1}(x)$ such that for $n>N_{1}, A_{n}(x)$ has density 1 at $x$. Then there is an $N_{2}>N_{1}$ such that for $n>N_{2}, x$ belongs to $E_{n}$. Thus $\cup_{1<n<\infty} E_{n}=E$. It remains only to show that $f$ is differentiable relative to $E_{n}$ and differentiates to $f_{\text {ap }}^{\prime}$. This is more involved.

Let $n$ be fixed and $x$ belong to $E_{n}$. Let $x_{k}$ be a sequence of points of $E_{n}$ converging to $x$. It will not hurt the generality of the argument to assume that $x=0$ and $f(0)=0$. Since $f$ is approximately differentiable at 0 there is a measurable set $V \subset Q$ having density 1 at 0 for which

$$
V-\lim _{x \rightarrow 0} \frac{f(x)}{x}=f_{\mathrm{ap}}^{\prime}(0) \text {. }
$$

By use of the Lusin-Menchoff Theorem [2], we may assume that $V$ is a perfect set. There is an $n^{-1}>\delta_{1}>0$ such that for all $0<x<\delta_{1}, m(V \cap$ $[0, x])>x 2^{-1}$. We assume that $x_{k}<\delta_{1}$ for all $k$ and define

$$
L_{k}=\left\{z: 0 \leqslant z<x_{k} \text { and } m\left(V \cap\left[z, x_{k}\right]\right) \geqslant 2^{-1}\left(x_{k}-z\right)\right\} .
$$

The set $L_{k}$ is not empty since 0 belongs to $L_{k}$. Let $l_{k}$ be the least upper bound of $L_{k}$. If $l_{k}=x_{k}$, select a point $z_{k}$ belonging to $L_{k}$ with $x_{k}>z_{k}>(1-$ $1 / k) x_{k}$. If $l_{k}<x_{k}$ let $z_{k}=l_{k}$. Then in either case

$$
m\left(V \cap\left[z_{k}, x_{k}\right]\right) \geqslant \frac{1}{2}\left(x_{k}-z_{k}\right) .
$$

For each $k x_{k}$ belongs to $E_{n}$, and since the length of $\left[z_{k}, x_{k}\right]$ is less than $n^{-1}$ we have

$$
m\left(A_{n}\left(x_{k}\right) \cap\left[z_{k}, x_{k}\right]\right) \geqslant \frac{3}{4}\left(x_{k}-z_{k}\right)
$$

Hence

$$
m\left(V \cap A_{n}\left(x_{k}\right) \cap\left[z_{k}, x_{k}\right]\right)>0 .
$$

We select another point $y_{k}$ from this intersection, strictly between $z_{k}$ and $x_{k}$. For the sequence $y_{k}$ we have

(i) $\lim _{k \rightarrow+\infty} f\left(y_{k}\right) / y_{k}=f_{\text {ap }}^{\prime}(0)$, and

(ii) $\left|f\left(y_{k}\right)-f\left(x_{k}\right)\right| \leqslant n\left(x_{k}-y_{k}\right)$. 
Now

$$
\frac{f\left(x_{k}\right)}{x_{k}}=\frac{f\left(x_{k}\right)-f\left(y_{k}\right)}{x_{k}-y_{k}}\left[1-\frac{y_{k}}{x_{k}}\right]+\frac{f\left(y_{k}\right)}{y_{k}} \cdot \frac{y_{k}}{x_{k}} .
$$

Thus from (i) and (ii) above if we show that $\lim _{k \rightarrow+\infty} y_{k} / x_{k}=1$, then $\lim _{k \rightarrow \infty} f\left(x_{k}\right) / x_{k}=f_{\text {ap }}^{\prime}(0)$. To get this final part we make a few preliminary observations. First we note that $z_{k}<y_{k}<x_{k}$, so it suffices to prove that $z_{k} / x_{k}$ approaches 1 . Next, the sequence $z_{k}$ was chosen in two different ways. For those $k$ such that $l_{k}=x_{k}$, we have $z_{k}>(1-1 / k) x_{k}$. Therefore, we need only consider any subsequence of $k$ 's for which $l_{k}<x_{k}$. In this case $z_{k}=l_{k}$, and we will be finished if we show that $\lim _{k \rightarrow+\infty} l_{k} / x_{k}=1$.

Let $0<\varepsilon<\frac{1}{4}$ be fixed. There is a $K$ such that for $k>K, m\left(V \cap\left[0, x_{k}\right]\right)$ $>(1-\varepsilon) x_{k}$. For such a $k$ consider the interval $\left[x_{k}-3 \varepsilon x_{k}, x_{k}\right]$. In this interval

$$
\begin{array}{r}
m\left(V \cap\left[x_{k}-3 \varepsilon x_{k}, x_{k}\right]\right) \geqslant x_{k}-\varepsilon x_{k}-\left[m\left(V \cap\left[0, x_{k}-3 \varepsilon x_{k}\right]\right)\right] \\
\geqslant x_{k}-\varepsilon x_{k}-\left[x_{k}-3 \varepsilon x_{k}\right]=2 \varepsilon x_{k}=\frac{2}{3} m\left(\left[x_{k}-3 \varepsilon x_{k}, x_{k}\right]\right) .
\end{array}
$$

Hence,

$$
l_{k} \geqslant x_{k}-3 \varepsilon x_{k}
$$

which completes the proof.

We note that Theorem 1 could be applied to get a version of the known result by Whitney [6]. However, we proceed to consider functions which are approximately differentiable everywhere in $[0,1]$.

THEOREM 2. If $f:[0,1] \rightarrow R$ has a finite approximate derivative, $f_{\mathrm{ap}}^{\prime}$, at every point of $[0,1]$, then there is a sequence of perfect sets $H_{n}$ and a sequence of differentiable functions $h_{n}$ such that

(i) $h_{n}(x)=f(x)$ over $H_{n}$,

(ii) $h_{n}^{\prime}(x)=f_{\text {ap }}^{\prime}(x)$ over $H_{n}$, and

(iii) $\cup_{1 \leqslant n<\infty} H_{n}=[0,1]$.

The sequence $\left(h_{n}, H_{n}\right)$ is called a decomposition of $f$. The corresponding sequence $\left(h_{n}^{\prime}, H_{n}\right)$ is the before-mentioned decomposition of the approximate derivative $f_{\mathrm{ap}}^{\prime}$.

Proof. We first obtain $H_{n}$. Let $E_{n}$ be the sets defined in Theorem 1. We have shown that $f$ differentiates to $f_{\text {ap }}^{\prime}$, relative to $E_{n}$. However, $E_{n}$ may have isolated points. We can express $E_{n}$ as the union of a perfect set $P_{n}$ and a countable set $C_{n}$. Let $C$ be the union, over $n$, of these $C_{n}$. Arrange the elements of $C$ into a sequence which we also label $x_{n}$. For each fixed $x_{n}$ we can find a perfect set $V_{n}$, having density 1 at $x_{n}$, such that

$$
V_{n}-\lim _{y \rightarrow x_{n}} \frac{f(y)-f(x)}{y-x}=f_{\text {ap }}^{\prime}\left(x_{n}\right) \text {. }
$$

We choose a disjoint sequence of intervals $I_{k}=\left[a_{k}, b_{k}\right]$ having the properties that

(1) the point $x_{n}$ is not in any $I_{k}$, 
(2) $\lim _{k \rightarrow+\infty} a_{k}=x=\lim _{k \rightarrow+\infty} b_{k}$, and

(3) $m\left(I_{k} \cap V_{n}\right)>0$ for each $k$.

For each $k$ it is possible to find an integer $N(k)$ such that $m\left(E_{N(k)} \cap I_{k} \cap\right.$ $\left.V_{n}\right)>0$. For such an $N(k)$ we select a perfect set $W_{k}$ from the intersection. Then we set $Q_{n}$ equal to the union, over $k$, of $W_{k}$ and $\left\{x_{n}\right\}$. Then $Q_{n}$ is perfect. Finally, we set $H_{n}=P_{n} \cup Q_{n}$. It is clear that the $H_{n}$ are perfect sets and, for each $x$ in $H_{n}$,

$$
H_{n}-\lim _{y \rightarrow x} \frac{f(y)-f(x)}{y-x}=f_{\mathrm{ap}}^{\prime}(x) .
$$

We note that the above argument could be applied in Theorem 1 to make $E_{n}$ perfect. At this point we are able to apply the theorem of Petruska and Laczkovich [3]. This theorem guarantees that for each $N$ it is possible to obtain a differentiable function $h_{n}$ such that $h_{n}=f$ over $H_{n}$. This completes the proof.

It is obvious that the existence of the sets $H_{n}$ presents a situation where the Baire category theorem can be usefully employed. We do so. As applications we present two corollaries. The first gives transparent proofs of two known theorems [1].

COROLlary 1. Let $f:[0,1] \rightarrow R$ have a finite approximate derivative $f_{\text {ap }}^{\prime}$ everywhere in $[0,1]$. Then

(a) there is a dense open set $U$ such that $f$ is differentiable on each component of $U$, and

(b) the function $f_{\text {ap }}^{\prime}$ is Baire 1 .

Proof. Let $\left(h_{n}, H_{n}\right)$ and $\left(h_{n}^{\prime}, H_{n}\right)$ be decompositions of $f$ and $f_{\text {ap. }}^{\prime}$.

(a) Let $H_{n}^{\circ}$ be the interior of $H_{n}$. Let $U$ be the union of the $H_{n}^{\circ}$. By the Baire category theorem, $U$ is a dense open subset of $[0,1]$. Clearly, since $f=h_{n}$ over $H_{n}^{\circ}, f$ is differentiable over every component of $U$.

(b) Let $P$ be a perfect set. Let $P_{n}=H_{n} \cap P$. Again an application of the Baire category theorem yields that there is an $N$ and $(a, b)$ such that $\varnothing \neq(a, b) \cap P \subset P_{N}$. Then $f_{\text {ap }}^{\prime}=h_{N}^{\prime}$ over $P_{N}$. Since $h_{N}^{\prime}$ is Baire 1 it has a point of relative continuity in $P_{N}$, and, hence, $f_{\text {ap }}^{\prime}$ is Baire 1 on $[0,1]$.

Basic to the concept of approximate differentiability is the idea that at a point $x_{0}$ we may disregard the behavior of a function over certain "small" sets. Therefore it becomes natural to expect that knowledge of the behavior of $f$ on a small set, such as nowhere dense sets of measure zero, would not - permit the prediction of the values of $f_{\text {ap }}^{\prime}$ over this set. However, the next corollary shows that this is not quite true.

Corollary 2. Let $f:[0,1] \rightarrow R$ and $g:[0,1] \rightarrow R$ be two measurable functions. Suppose $P$ is any perfect set such that $f(x)=g(x)$ over $P$. Suppose, in addition, that at every point of $P f$ is approximately differentiable and $g$ is differentiable. Then there is an open interval $(a, b)$ with $(a, b) \cap P \neq \varnothing$ such that $f_{\text {ap }}^{\prime}=g^{\prime}$ at every point of $(a, b) \cap P$. 
Proof. Let $h(x)=f(x)-g(x)$ for all $x$ in $[0,1]$. Then over $P h=0$ and $h_{\mathrm{ap}}^{\prime}=f_{\mathrm{ap}}^{\prime}-g^{\prime}$. By Theorem 1 there is a sequence of closed sets $P_{n}$ such that $h$ differentiates to $h_{\text {ap }}^{\prime}$ over $P$ and $\cup_{1 \leqslant n<\infty} P_{n}=P$. Once again the Baire category theorem yields an $(a, b)$ and $N$ such that $\varnothing \neq(a, b) \cap P \subset P_{N}$. Now $P$ is perfect, and for every $x$ in $(a, b) \cap P$ we have

$$
P-\lim _{y \rightarrow x} \frac{h(y)-h(x)}{y-x}=h_{\text {ap }}^{\prime}(x)=0=f_{\text {ap }}^{\prime}(x)-g^{\prime}(x) .
$$

ACKNOWLEDGMENTS. The author appreciates valuable discussions he had with Professor C. E. Weil which led to the development of this paper.

\section{REFERENCES}

1. C. Goffman and C. J. Neugebauer, On approximate derivatives, Proc. Amer. Math. Soc. 11 (1960), 962-966.

2. C. Goffman, C. J. Neugebauer and T. Nishiura, Density topology and approximate continuity, Duke Math. J. 28 (1961), 497-505.

3. M. Laczkovich and G. Petruska, Baire 1 functions, approximately continuous functions and derivatives, Acta. Math. Acad. Sci. Hungar. 25 (1974), 189-212.

4. R. J. O'Malley, A density property and applications, Trans. Amer. Math. Soc. 199 (1974), 75-87.

5. G. Tolstoff, Sur la derive approximative exacte, Mat. Sb. 4 (1938), 499-504.

6. H. Whitney, On totally differentiable and smooth functions, Pacific J. Math. 1 (1951), 143-159.

Department of Mathematics, University of Wisconsin, MinwaukeE, Wisconsin 53201 